Rakenteiden Mekaniikka (Journal of Structural Mechanics)

Vol. 51, No 4, 2018, pp. 20-37

http://rakenteidenmekaniikka.journal.fi/index

https://doi.org/10.23998/rm.70292

(C)Author(s) 2018.

Open access under CC BY-SA 4.0 license.

\title{
Stress concentration factor for interacting surface notch and subsurface hole
}

\author{
Silas Z. Gebrehiwot ${ }^{1}$, Heikki Remes and Anssi T. Karttunen
}

\begin{abstract}
Summary. We propose a conservative method for the calculation of the maximum stress concentration factor (SCF) for an interacting notch-hole pair and for a double semi-circular notch (i.e., a notch that has an additional small semi-circular notch ahead of its tip). The method is based on a linearly elastic Airy stress function solution for a circular hole. The notch-hole and double notch configurations are aligned vertically with respect to uniform uniaxial (horizontal) stress. This means, a uniform horizontal tension is applied to a notch-hole pair that lie on a vertical axis. For the notchhole pair, the maximum interacting SCFs are calculated for edge to edge gaps equal to hole sizes of $2.5 a, 5 a, 10 a$ and $15 a$, where $a$ is the hole radius. The analytical results are validated by 2-D finite element calculations. The presented simple approach provides good results with errors well below $10 \%$ in most cases compared to the detailed finite element analyses. Fatigue notch factors that can be thought of as the effective SCFs in fatigue analyses are determined. By using the simple approach, computationally costly finite element analysis can be avoided.
\end{abstract}

Keywords: stress function, semi-circular notch, subsurface hole, stress concentration factor, interaction

Received 30 April 2018. Accepted 15 October 2018. Published online 31 December 2018.

\section{Introduction}

Machineries, vehicles, ships and airliners have numerous components which contain stress concentrations. In Peterson's book [1], stress concentration is defined as a localized phenomenon of higher stress near geometrical changes that is beyond the nominal stress value. Geometrical discontinuities occur in many forms such as surface notches or subsurface defects, e.g., circular or elliptical porosities or inclusions. The discontinuities can reduce the

${ }^{1}$ Corresponding author: silas.gebrehiwot@ arcada.fi 
fatigue life of components considerably. To avoid fatigue failure related to stress concentrations, detailed understanding of them in engineering design is required.

In addition to an isolated discontinuity, the interaction of neighbouring geometrical discontinuities can have a severe effect on the fatigue life of a structural component. The interaction effect is defined as an increase or decrease of stress concentration when notches and holes exist close to each other. The interaction is affected by the relative sizes of the discontinuities and by their orientations with respect to loading. Murakami conducted a number of SCF studies for horizontal arrays of semi-circular, semi-elliptical, periodical Vshaped notches and holes [2]. For an array (or line) of notches and holes parallel to a tensile load, an interaction occurs that decreases stress concentrations. On the other hand, an increase in stress concentration tends to occur when notch and hole arrays are aligned perpendicular to tensile loading. The fatigue life of a component is reduced under such conditions [1], [3].

There are several other studies on the interaction of neighbouring geometrical discontinuities based on analytical, experimental and finite element simulation approaches in addition to Murakami's work [2]. Analytical solutions for a plate containing two holes under a uniaxial and biaxial stresses were proposed by Ling and Haddon for a variety of hole-hole orientations [1], [3]. The studies focused on subsurface holes and showed the effect of relative orientations and hole sizes on the SCF. With two holes existing close to each other under uniaxial or biaxial loading, the stress distribution is clearly different from that of a single hole. A case of two circular holes in an infinite plate was also studied by Hamad, Masuda and Mizushima [4]. They used a numerical approach to estimate an interacting SCF. Their results underestimated the SCF compared to the works by Ling, Haddon and Ishida [3], [4]. The stress amplification between two holes under tension was modelled by $\mathrm{Wu}$ and Markenskoff [5]. The amplification between the holes was expressed in terms of the holes' edge to edge distance. However, the method has problems with singularities when the edge to edge distance approaches zero. Whitely studied an open hole placed on a curve radius [6].

Subsurface and close to surface porosities have been investigated experimentally and considered as sources of fatigue failures and sites of crack initiation by several authors [710]. Reed and Miller assessed the fatigue behaviour of turbine materials at elevated temperatures in air and vacuum environments. Their study revealed that crack initiations occur in subsurface pores which are close to surface notches [7]. Furnish et al. studied the stress concentration and notch sensitivity of nanocrystalline NiFe under tension-tension fatigue test [8]. The investigation showed that for over 10000 loading cycles, a crack initiates right beneath a semi-circular notch micromachined by dual-beam focused ion beam (FIB). The interaction effect of pores has been studied experimentally and verified by finite element models as a pore clustering effect [9]. It was shown that the stress concentration increases sharply for two pores aligned perpendicular to a uniaxial load. In a similar experimental study, clusters of pores have been identified as crack initiation sites for specimens with double edge notches [10]. On average, the fatigue life of the notched specimens was reduced by $20 \%$ in comparison to smooth specimens.

This paper investigates the maximum (interacting) SCF of neighbouring notches and holes and double semi-circular notches. A conservative approach is proposed to calculate the 
maximum interacting SCF for notch-hole gaps of different sizes. The approach is based on an analytical solution for a hole in an infinite plate. The solution is used for semi-infinite plates with vertically aligned notches and holes under a uniaxial loading in horizontal direction. The approach is validated by finite element simulations. The study includes notchhole and notch-notch geometries with 5:1 and 10:1 radii ratios.

\section{Stress solution for an infinite plate with a hole}

The 2-D stress equilibrium equations for a linearly elastic solid in polar coordinates are

$$
\begin{gathered}
\frac{\partial \sigma_{r}}{\partial r}+\frac{1}{r} \frac{\partial \tau_{r \theta}}{\partial \theta}+\frac{1}{r}\left(\sigma_{r}-\sigma_{\theta}\right)=0, \\
\frac{\partial \tau_{r \theta}}{\partial r}+\frac{1}{r} \frac{\partial \sigma_{\theta}}{\partial \theta}+\frac{2 \tau_{r \theta}}{r}=0 .
\end{gathered}
$$

To determine stress solutions for two-dimensional problems, it is convenient to use the Airy stress function $\Phi(r, \theta)$ so that

$$
\sigma_{r}=\frac{1}{r} \frac{\partial \Phi}{\partial r}+\frac{1}{r^{2}} \frac{\partial^{2} \Phi}{\partial \theta^{2}}, \quad \sigma_{\theta}=\frac{\partial^{2} \Phi}{\partial r^{2}}, \quad \tau_{r \theta}=-\frac{1}{r} \frac{\partial^{2} \Phi}{\partial r \partial \theta}+\frac{1}{r^{2}} \frac{\partial \Phi}{\partial \theta}
$$

which satisfy the equilibrium equations (1). To ensure compatibility, it is required that the stress function satisfies the governing equation

$$
\left(\frac{\partial^{2}}{\partial r^{2}}+\frac{1}{r} \frac{\partial}{\partial r}+\frac{1}{r^{2}} \frac{\partial^{2}}{\partial \theta^{2}}\right)\left(\frac{\partial^{2} \Phi}{\partial r^{2}}+\frac{1}{r} \frac{\partial \Phi}{\partial r}+\frac{1}{r^{2}} \frac{\partial^{2} \Phi}{\partial \theta^{2}}\right)=0
$$

Solutions to elasticity problems in polar coordinates are obtained by finding a solution of equation (3) that satisfies the stress boundary conditions of the 2-D structure at hand. An infinite plate with a hole under uniaxial tension is shown in Figure 1. The boundary conditions for the plate are [11]

$$
\begin{gathered}
\sigma_{r}=\tau_{r \theta}=0 ; r=a, \\
\sigma_{x} \rightarrow \sigma ; r \rightarrow \infty, \\
\sigma_{y}, \tau_{x y} \rightarrow 0 ; r \rightarrow \infty .
\end{gathered}
$$




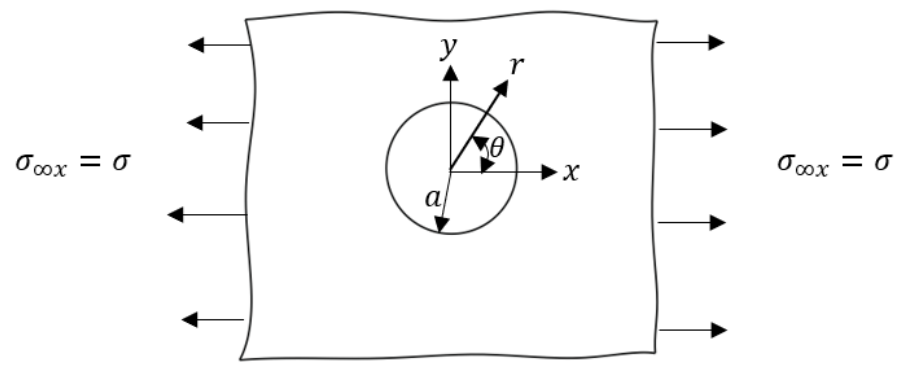

Figure 1. Hole in an infinite plate under uniaxial tension.

In polar coordinates, the far-field conditions become [11]

$$
\begin{gathered}
\sigma_{\mathrm{r}}=(\sigma / 2)(1+\cos 2 \theta) ; r \rightarrow \infty, \\
\sigma_{\theta}=(\sigma / 2)(1-\cos 2 \theta) ; r \rightarrow \infty, \\
\tau_{\mathrm{r} \theta}=-(\sigma / 2)(\sin 2 \theta) ; r \rightarrow \infty .
\end{gathered}
$$

The Airy stress function that satisfies the boundary conditions and the governing equation (3) reads as

$$
\Phi=\frac{1}{4} \sigma r^{2}(1-\cos 2 \theta)+A \ln r+B \theta+C \cos 2 \theta+\frac{D}{r^{2}} \cos 2 \theta
$$

where $A, B, C$ and $D$ are solved from the boundary conditions [Eq. (4)]. The stresses are $[11,12]$

$$
\begin{gathered}
\sigma_{r}=\frac{\sigma}{2}\left[1-\left(\frac{a}{r}\right)^{2}\right]+\frac{\sigma \cos 2 \theta}{2}\left[1-4\left(\frac{a}{r}\right)^{2}+3\left(\frac{a}{r}\right)^{4}\right] \\
\sigma_{\theta}=\frac{\sigma}{2}\left[1+\left(\frac{a}{r}\right)^{2}\right]-\frac{\sigma \cos 2 \theta}{2}\left[1+3\left(\frac{a}{r}\right)^{4}\right] \\
\tau_{r \theta}=-\frac{\sigma \sin 2 \theta}{2}\left[1+2\left(\frac{a}{r}\right)^{2}-3\left(\frac{a}{r}\right)^{4}\right] .
\end{gathered}
$$

The stresses are displayed in Figure 2. Only the hoop stress $\sigma_{\theta}$ is nonzero on the edge of the hole and the maximum $\mathrm{SCF}=\sigma_{\theta} / \sigma=3$ occurs at angles of $90^{\circ}(\pi / 2)$ and $270^{\circ}(3 \pi / 2)$. 

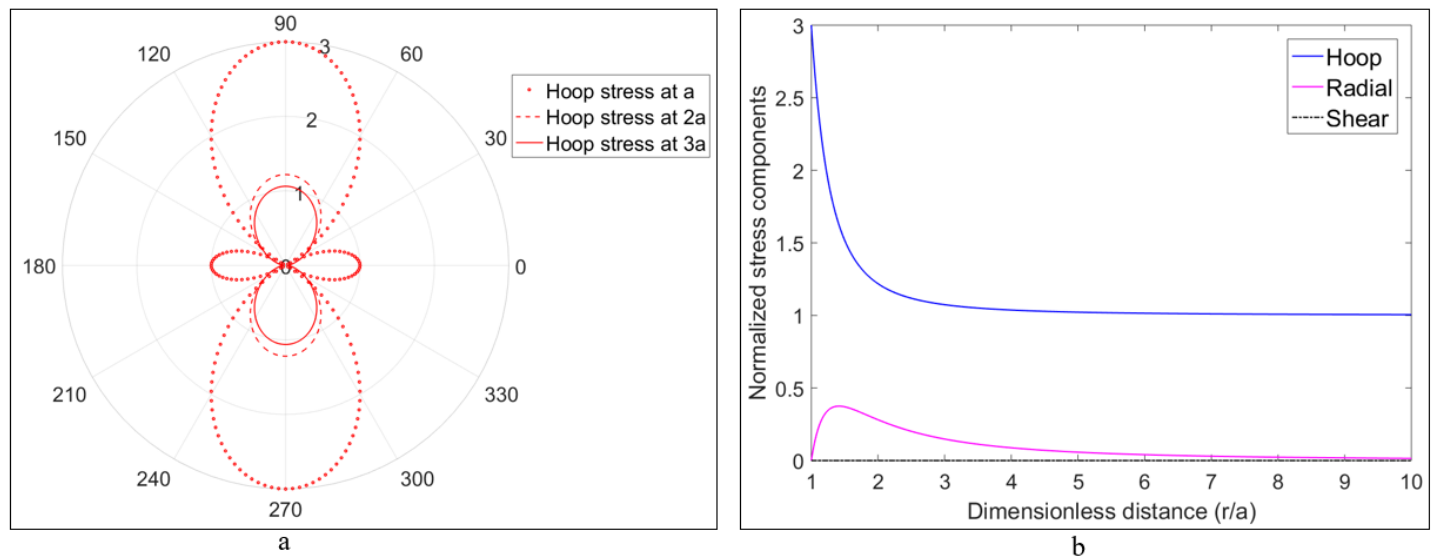

Figure 2. a) Dimensionless hoop stresses $\sigma_{\theta} / \sigma$ around a circular hole. b) Distributions of radial, shear, hoop stresses normalized by nominal remote stress at $90^{\circ}(\pi / 2)$ and $270^{\circ}(3 \pi / 2)$.

\section{Conservative estimation approach}

According to Maunsell [13], the hoop stress distribution of a semi-circular profile likens that of a circular one. In this paper, we assume that the semi-circular profile has the same hoop stress distribution as a circular profile at angles $90^{\circ}$ and $270^{\circ}$ (Fig. 2). However, the maximum $\mathrm{SCF}$ on the edge of a semi-circular profile is known to be slightly higher than that of a circular one ( $\mathrm{SCF}=3$ for circular). In this paper, the maximum $\mathrm{SCF}=3.065$ at $270^{\circ}(3 \pi / 2)$ will be used for the surface notch whenever required. The value is chosen as it is the result of exact calculation by Ling in 1948 and later by M.Ishida in 1953 [2], [3].

Figures 3(a) and 3(b) show a double semi-circular notch and a notch-hole pair, respectively. The conservative approach uses the hoop stress component of the semi-circular surface notch (A) as a remote tensile stress for the notch or hole (B) beneath it; i.e. the smaller radii notch or hole (B) is within the stress field generated by the larger radius notch (A). Figures 4(a) and 4(b) show that we use the hoop stress component of the notch (A) at the top of the edge $(\pi / 2)$ of the small hole as an approximation for a uniform stress field. Similarly, the hoop stress component of the notch (A) is used at the bottom of the smaller notch (B) for the double notch geometry. The method is called conservative because the hoop stress $\sigma_{\theta}$ at $r=r_{1}$ provides the highest possible far-field stress for the small hole (Fig. 4(b)), and because it will turn out that the method provides slightly higher stress concentration factors than detailed finite element calculations. Similar reasoning holds for the double notch geometry. Here, $r_{1}$ is the distance between the centre of the notch and the top edge of circular hole at $(\pi / 2)$, and $r_{2}$ from the centre of the surface notch to the bottom of the smaller notch at $(3 \pi / 2)$, (Fig. 3(a) and 3(b)). 

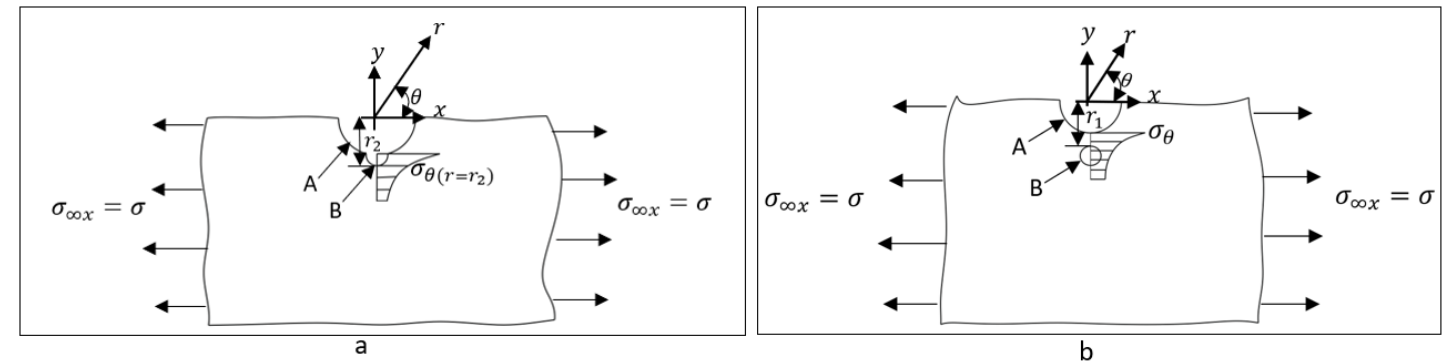

Figure 3. Hoop stress distribution of the large semi-circular surface notch is assumed to be similar to that of a circular hole. a) Double semi-circular notch b) Notch-hole configuration.
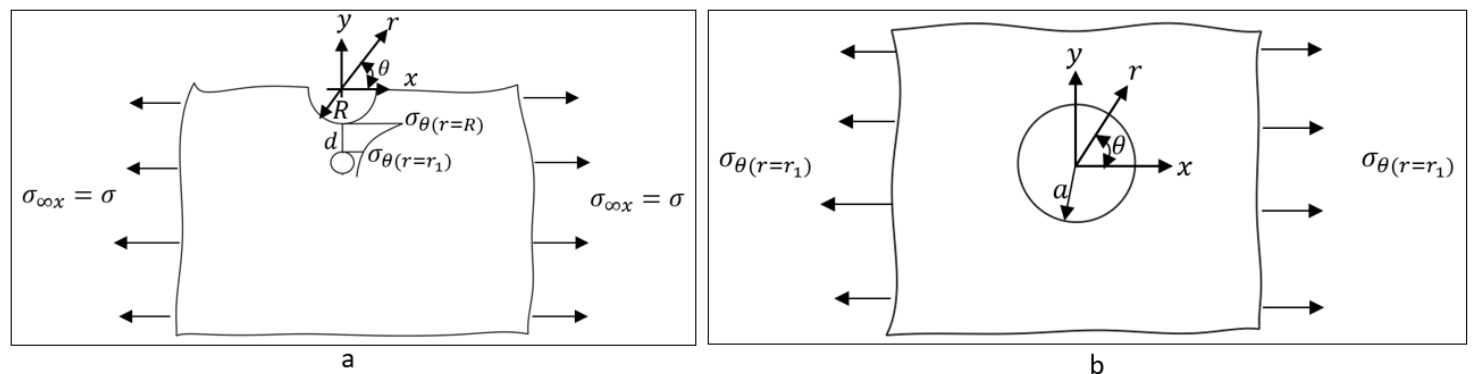

Figure 4. a) Conservative SCF estimation uses the hoop stress component of the semi-circular notch for the small hole ( $\mathrm{R}$ is the notch radius). b) The hoop stress component $\sigma_{\theta\left(r=r_{1}\right)}$ is taken to represent a uniform stress field.

In general, the expression for a SCF is presented as a ratio between the maximum stress and the nominal stress [1]

$$
\mathrm{k}_{\mathrm{t}}=\frac{\sigma_{\mathrm{max}}}{\sigma_{\mathrm{nom}}} .
$$

Using Eq. (8), the maximum stress is now calculated for the small hole (Fig. 4(b)) by employing the surface notch stress $\sigma_{\theta}$ at $r=r_{1}$ as the nominal stress

$$
\sigma_{\max }=\mathrm{k}_{\mathrm{t}(\text { hole })} * \sigma_{\theta\left(\mathrm{r}=\mathrm{r}_{1}\right)} \text {. }
$$

The conservative SCF of the interacting notch and hole pair is then estimated using the original far field nominal stress $\sigma$ in Eq. (8)

$$
k_{t(C)}=\frac{\mathrm{k}_{\mathrm{t}(\text { hole })} * \sigma_{\theta\left(\mathrm{r}=\mathrm{r}_{1}\right)}}{\sigma} .
$$


The SCF for double semi-circular notches is also determined using Eq. (10). However, SCF of the hole, $\mathrm{k}_{\mathrm{t}(\text { hole })}=3$ should be replaced by $\mathrm{k}_{\mathrm{t}(\text { notch })}=3.065$ and $\mathrm{r}=\mathrm{r}_{2}$ is the notch bottom (Fig. 3(a)).

The material-dependent fatigue notch factor $k_{f}$ can be thought of as the effective SCF in fatigue analyses. The relation between $k_{f}$ and the usual $k_{t}$ is expressed by the notch sensitivity [3]

$$
q=\frac{k_{f}-1}{k_{t}-1}
$$

In Eq. (11), a notch sensitivity value of $q=1$ indicates that a material is highly sensitive to a notch and occurs when $k_{f}=k_{t}$. A notch sensitivity of zero indicates that a material is not sensitive to notches and occurs when $k_{f}=1$. A notch sensitivity expression as a function of the notch radius $\rho$ and a material constant $\alpha$ [1], [3] is

$$
q=\frac{1}{1+\frac{\alpha}{\rho}} .
$$

From Equations (11) and (12), the fatigue notch factor can be determined by

$$
k_{f}=\left[\left(\frac{1}{1+\frac{\alpha}{\rho}}\right)\left(k_{t}-1\right)+1\right] \text {. }
$$

The material constant $\alpha$ is known for a variety of steels [1]. Based on the available data, $k_{f}$ can be calculated using Eq. (13). For typical low, medium and high ultimate tensile strength values of $344.7 \mathrm{MPa}, 689.5 \mathrm{MPa}$ and $1723.7 \mathrm{MPa}$, respectively, the material constants are $\alpha_{\text {low }}=0.381 \mathrm{~mm}, \alpha_{\text {medium }}=0.1778 \mathrm{~mm}$ and $\alpha_{\text {high }}=0.03302 \mathrm{~mm}$, respectively. The notch radius, $\rho$ refers to the root notch radius and radius of the smaller hole for the doublenotch and notch-hole geometries, respectively.

\section{Results}

The conservative SCF estimations were made using Eq. (10). The maximum SCF and stress distributions of interacting notches and holes were also analyzed by finite element simulations for validation purposes. For the finite element analyses, 4-node quadrilateral bilinear CPS4 plane stress elements were used in Abaqus finite element software. Table 1 presents the maximum interacting SCF estimations by both methods.

The double semi-circular notches and notch-hole pairs have different profiles. Therefore, the distance $d$ between geometries (notch-notch and notch-hole) is considered accordingly. In the double semi-circular notch, the bigger notch has a smaller one ahead of its tip. Geometrically, they are joined and the midpoint on the smaller semi-circular notch is located at bottom edge of the bigger one. Therefore, the distance $d$ between the two notches is zero. 
However, for the notch-hole pairs, the distance $d$ represents edge to edge gaps between the notches and holes. It is measured vertically from the bottom edge of the bigger radii notches to the top edge of the holes.

Table 1. Comparisons of interacting SCF. See Figs. 5 and 6 for the studied geometries.

\begin{tabular}{|c|c|c|c|c|c|}
\hline \multirow{3}{*}{$\begin{array}{l}\text { Radii } \\
\text { ratio } \\
(R / a)\end{array}$} & \multirow{3}{*}{$\begin{array}{c}\text { Distance } d \\
\text { ( } a \text { is hole } \\
\text { radius) }\end{array}$} & \multicolumn{4}{|c|}{ Maximum interacting stress concentration factors (SCF) } \\
\hline & & $k_{t(\mathrm{FEM})}$ & $k_{t(\mathrm{C})}$ & & Note \\
\hline & & Value & Value & Error $(\%)$ & \\
\hline 5 & 0 & 6.21 & 6.35 & 2.20 & Double notch \\
\hline 5 & $2.5 a$ & 4.21 & 4.56 & 8.21 & Notch-hole \\
\hline 5 & $5 a$ & 3.31 & 3.66 & 10.39 & Notch-hole \\
\hline 5 & $10 a$ & 3.07 & 3.11 & 1.46 & No interaction \\
\hline 10 & 0 & 7.39 & 7.47 & 1.10 & Double notch \\
\hline 10 & $2.5 a$ & 5.63 & 5.80 & 3.02 & Notch-hole \\
\hline 10 & $5 a$ & 4.22 & 4.55 & 7.90 & Notch-hole \\
\hline 10 & $10 a$ & 3.35 & 3.66 & 9.04 & Notch-hole \\
\hline 10 & $15 a$ & 3.10 & 3.36 & 8.30 & Notch-hole \\
\hline
\end{tabular}

The maximum SCFs and their locations are identified using both approaches. For the double-notch configurations, the small semi-circular notch has the maximum SCF at $270^{\circ}$ $(3 \pi / 2)$. Similarly, the small hole has the maximum SCF at its top edge $(\pi / 2)$ for the notchhole pairs. The interacting effect of the SCF vanishes at a distance of $10 a$ for the 5:1 radii ratio. However, for the 10:1, the interacting effect exists at $15 a$. This is due to the notch effect [2]. The stress distributions of geometrically similar notches depend on notch size. The bigger notch has higher stress distribution than that of the smaller one [2]. Therefore, the notch with 10:1 radii ratio has higher hoop stress distribution than the 5:1. This makes the 10:1 notchhole pair have the interacting effect of the SCF at 15a. For the 5:1 notch-hole pair, the hoop stress distribution reduces to the nominal remote tension at $10 a$. Therefore, the SCF for the notch and hole can be calculated individually.

The finite element results are presented in Figures 5 and 6, respectively. Coordinate points along a vertical axis are used to plot the stress distributions. In Figures 5 and 6, the graphs are discontinuous at the hole locations. Figure 5 shows the normalized stress distributions of the 5:1 notch-hole pairs at $2.5 a, 5 a$, and $10 a$ edge to edge gaps. Similarly, the normalized stress distributions of the notch-hole pairs with 10:1 radii ratio are analyzed in Figure 6. 


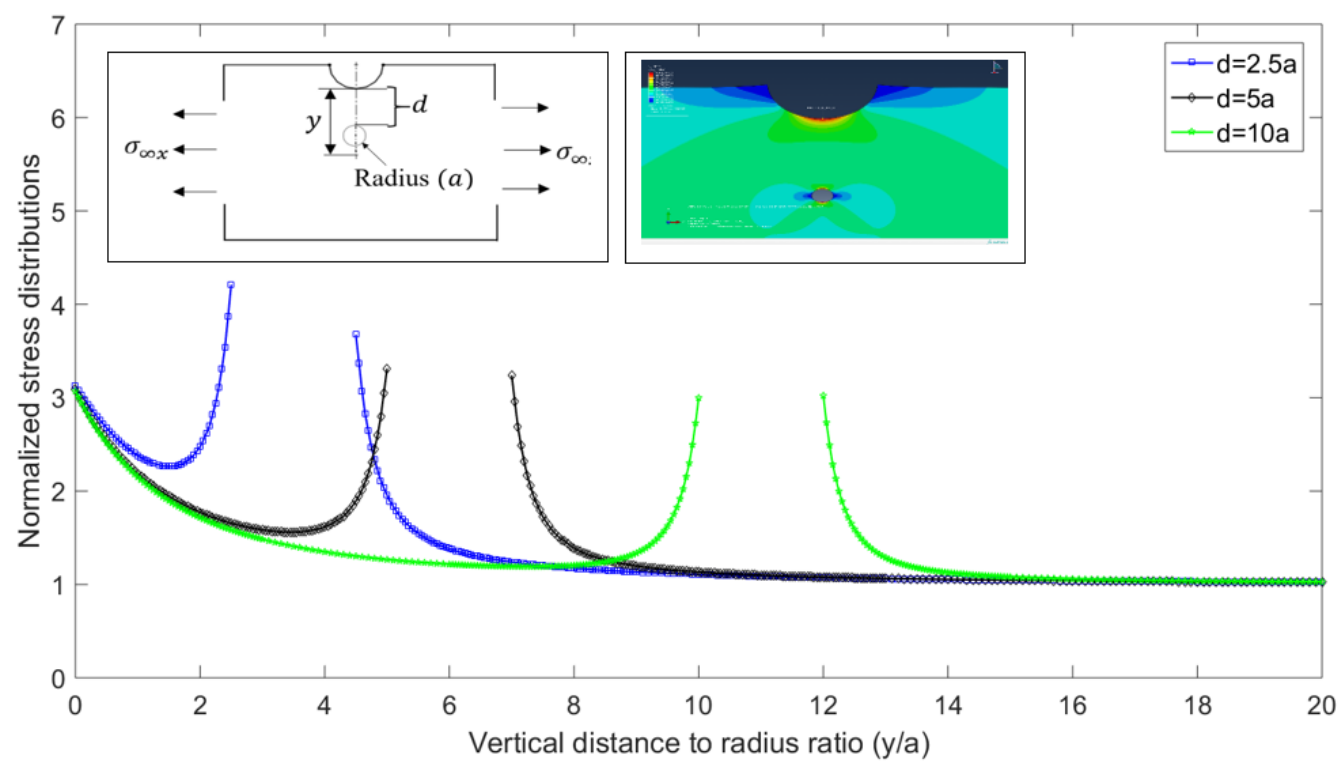

Figure 5. Normalized stress distributions for notch-hole pairs at gaps of $d=2.5 a, 5 a$, and $10 a(R / a=$ $5)$ by finite element calculations. The curve discontinuities occur at the locations of the subsurface holes. $y$ is the distance measured vertically from the bottom edge of the notch.

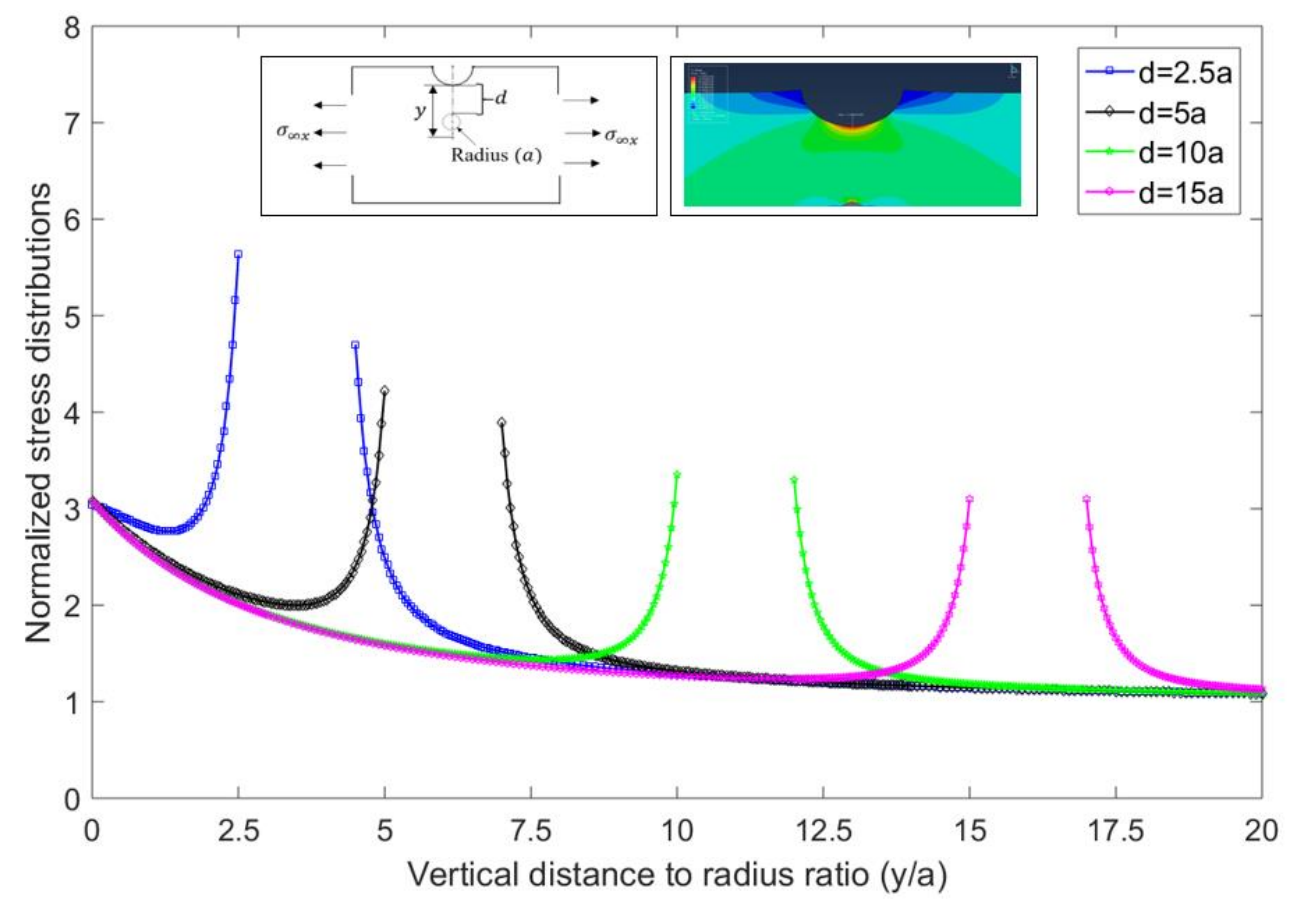

Figure 6. Normalized stress distributions for notch-hole pairs at gaps of $d=2.5 a, 5 a, 10 a$ and $15 a$ $(R / a=10)$ by finite element calculations. Curve discontinuities occur at the locations of the subsurface holes. $y$ is the distance measured vertically from the bottom edge of the notch. 
From Table 1, the interacting SCF estimations for the notch-hole pairs are further demonstrated by Figures 7 and 8. Figures 7 and 8 clearly indicate the estimations graphically. The conservative approach estimates $\mathrm{k}_{\mathrm{t}}=7.47$ for the double semi-circular notch of 10:1 radii ratio. This estimation is better than that of Murakami's [2] where $\mathrm{k}_{\mathrm{t}}=8.16$.

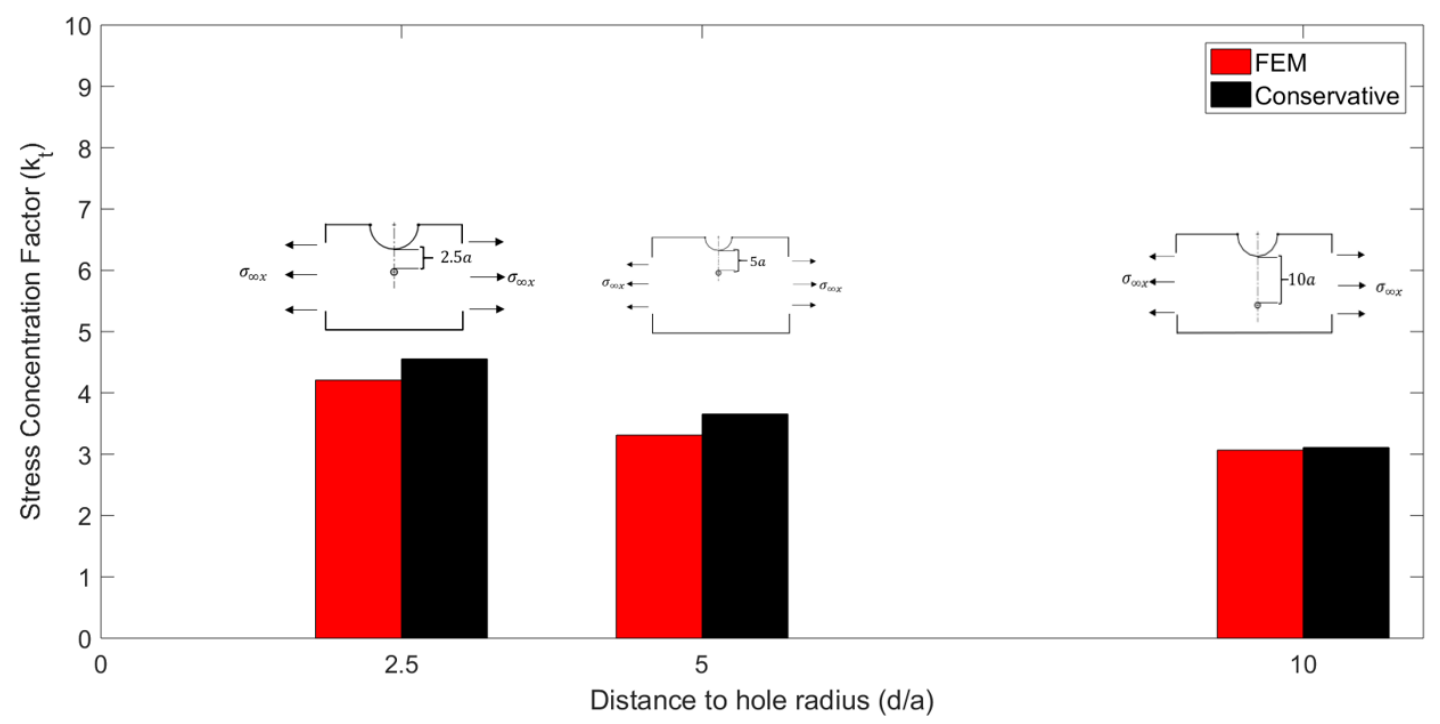

Figure 7. Interacting SCF analyses using theoretical and finite element simulation for the 5:1 radii ratio. SCF estimations are made for notch-hole pairs at gaps of $d=2.5 a, 5 a$, and $10 a$

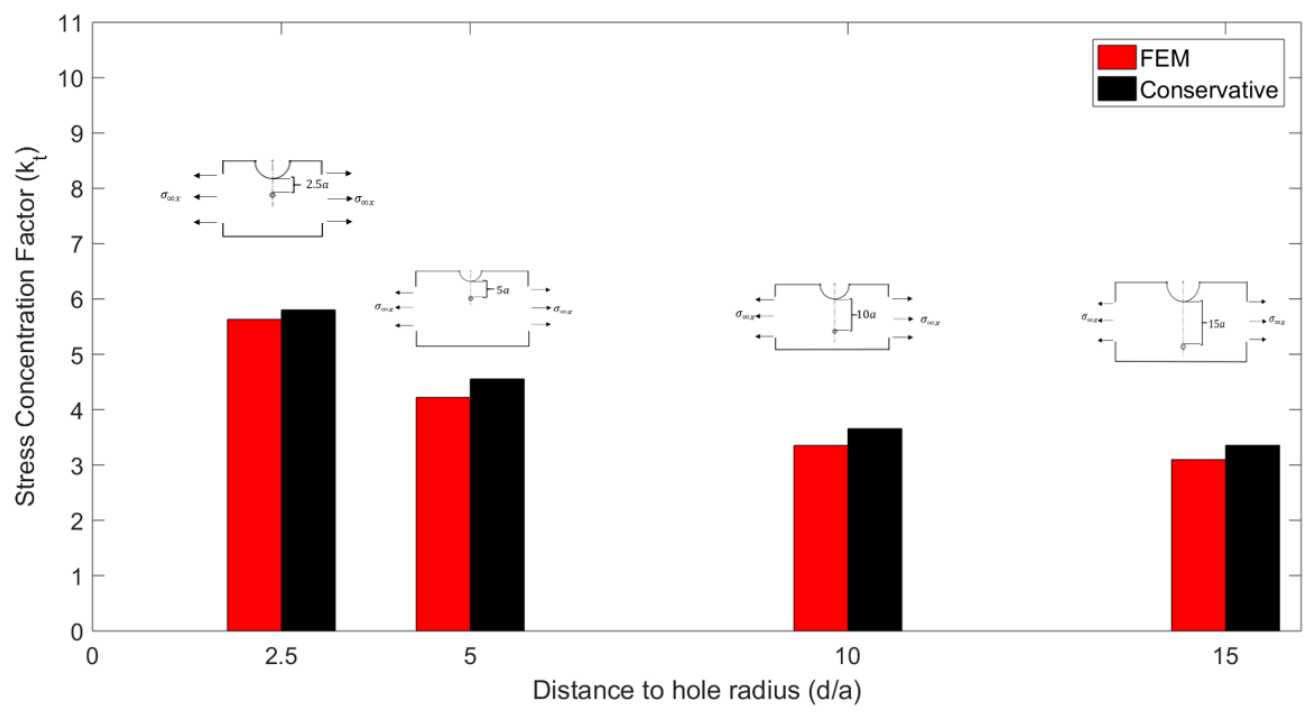

Figure 8. Interacting SCF analyses using theoretical approaches and finite element simulation for the10:1 radii ratio. SCF estimations are made for notch-hole pairs at gaps of $d=2.5 a, 5 a, 10 a$ and $15 a$ 
The results given by the conservative approach are further utilized to estimate the fatigue notch factors of low, medium and high ultimate tensile strength steels. As discussed, the fatigue notch factor is influenced by material's ultimate tensile strength [1]. Figures 9 and 10 present the fatigue notch factors for the notch-hole pairs.

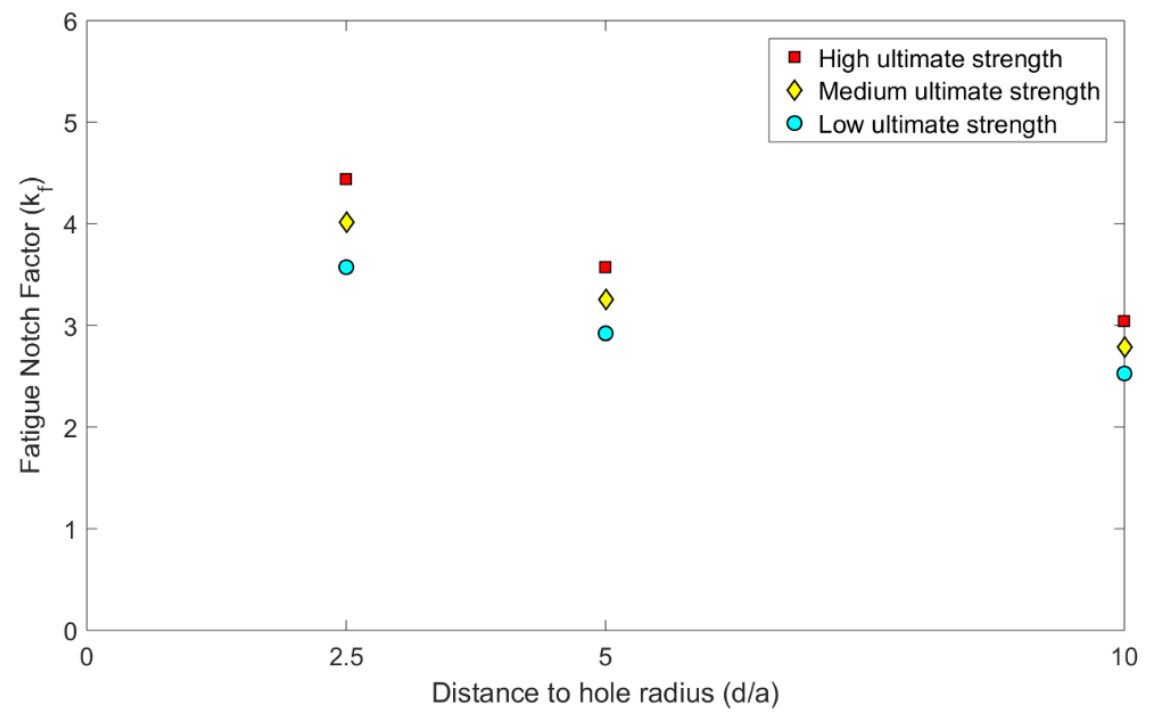

Figure 9. Fatigue notch factors of low, medium and high tensile ultimate strength for 5:1 radii ratio. Fatigue notch factor estimations are made for notch-hole pairs at gaps of $d=2.5 a, 5 a$, and $10 a$.

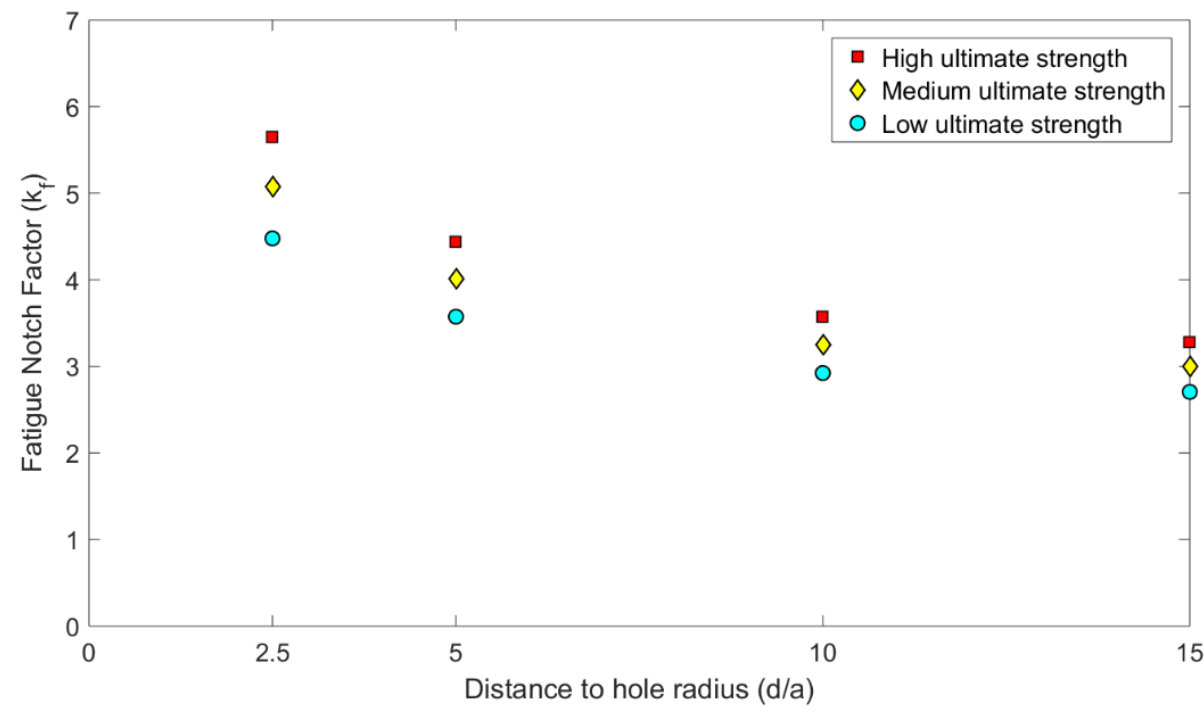

Figure 10. Fatigue notch factors of low, medium and high tensile ultimate strength for 10:1 radii ratio. Fatigue notch factor estimations are made for notch-hole pairs at gaps of $d=2.5 a, 5 a$, $10 a$, and $15 a$. 


\section{Conclusions}

Maximum SCFs of interacting notches and holes in semi-infinite plates oriented perpendicular to uniaxial tension were estimated. The analyses considered surface notch-hole pair radii ratios of 10:1 and 5:1 for different notch-hole gap sizes $d$. The results by the developed simple theoretical approach were validated by 2-D finite element analyses. The conservative approach overestimated the SCF by $1.1-10.39 \%$ compared to finite element analyses depending on the geometry.

The theoretical and the finite element analyses showed that the subsurface holes exhibit higher stress concentrations than the surface notches for which SCF $\approx 3$. This is in agreement with the experimental findings that subsurface pores are the sites of crack initiations [7] [10]. It was also observed that, for the 5:1 radii ratio, the interaction effect of SCF vanishes at notch-hole gap of $10 a$ and above. In the same manner, for 10:1 radii ratio, the interaction vanishes at notch-hole gap of $20 a$ and above. We note that the study was limited to double semi-circular notches and notch-hole models with edge to edge gap sizes $d \geq 2.5 a$. For gap sizes $0<d<2.5 a$, the interacting SCF generally increases. Ling and Haddon used mathematical analyses to determine the SCF for two circular holes on infinite plates [1], [3]. The SCF goes to infinity as the gap approaches to zero. A similar trend is expected for the notch-hole pair gaps between 0 and $2.5 a$.

The presented approach is used to estimate SCF of notch-hole pair with the notch radius, $R$ larger than that of the hole, $a$. This approach can also be utilized to estimate interacting SCF when $a>R$. In this case, the notch will be within the stress field of the larger radius hole.

Considering the interaction of stress concentrations, the fatigue notch factors of three different steel materials were estimated. Figures 9 and 10 indicate that with the increase in the material ultimate strength the notch sensitivity increases. Therefore, the steel with the highest ultimate strength has the lowest endurance limit.

\section{References}

[1] R.E. Peterson. Stress Concentration Factors. New York: John Wiley \& Sons, 1974, pp. 20-26.

[2] Y. Murakami. Theory of Elasticity and Stress Concentration. Chichester, John Wiley \& Sons, 2017, Part II, Ch. 1 pp. 248-270, Ch. 4 pp. 319-332, Ch. 5 pp. 335-337.

[3] W.D. Pilkey and D.F. Pilkey. Peterson's Stress Concentration Factors, 3rd ed. New Jersey: John Wiley \& Sons, 2008, Ch. 1, pp. 3-24, Ch. 2 pp. 58-68, Ch. 4 pp. 178-308.

[4] M. Hamada, I. Mizushima and T. Masuda. On the stress concentration in a strip with two circular holes subjected to tension, Bulletin of JSME, July 1980, Vol. 23, no. 181, pp. 1043-1047. https://doi.org/10.1299/jsme1958.23.1043

[5] L. Wu, X. Markenscoff. Singular stress amplification between two holes in tension. Journal of Elasticity, 1996, Vol. 44, pp. 143. https://doi.org/10.1007/BF00042474 
[6] D.W. Whitely. Interacting stress concentration factors and their effect on fatigue of metallic aero structures: previous studies of interacting stress concentrations. Doctoral Dissertations, Missouri University of Science \& Technology, 2013, pp. 15-32.

[7] P.A.S. Reed and M.D. Reed. Comparison of low cycle (notch) fatigue behaviour at temperature in single crystal turbine blade material. Proc. of the 11th int. symposium on superalloys. Southampton, United Kingdom, Sept. 2008, pp. 527-533. https://doi.org/10.7449/2008/Superalloys_2008_527_533

[8] T.A. Furnish et al. Fatigue stress concentration and notch sensitivity in nanocrystalline metals. Journal of Material Research, Mar. 2016, Vol. 31, pp. 740-752. https://doi.org/10.1557/jmr.2016.66

[9] Z. Xu, W. Wen and T. Zhia. Effects of pore position in depth on stress/strain concentration and fatigue crack Initiation. The Minerals, Metals \& Materials Society and ASM International, Aug. 2012, Vol. 43, pp. 2763-2770. https://doi.org/10.1007/s11661-011-0947-x

[10] A. H. Rosenberg et al. The variability of fatigue in notched bars of IN100. Proc. of the 12th int. symposium on superalloys. Universal Technology Corporation, Dayton, USA, Oct. 2012. https://doi.org/10.1002/9781118516430.ch16

[11] M.H. Sadd. Elasticity: Theory Application and Numerics, 2nd ed. Burlington, Elsevier, 2009, pp. 5-71.

[12] J.R. Barber. Elasticity: Solid Mechanics and its Applications, 2nd ed. Dordrecht, Kluwer Academic Publishers, 2002, pp. 34-108.

[13] F.G. Maunsell. Stresses in a notched plate under tension. Phil. Mag., 1936, Vol. 21, pp. 765-773. https://doi.org/10.1080/14786443608561624

Silas Z. Gebrehiwot

Aalto University, Department of Mechanical Engineering

P.O. Box 11000, FI-00076 AALTO

Arcada University of Applied Sciences

Jan-Magnus Janssonin aukio 1, 00550 Helsinki, Finland

Häme University of Applied Sciences (HAMK)

Hämeenlinna, Finland

silas.gebrehiwot@arcada.fi

Heikki Remes, Anssi T. Karttunen

Aalto University, Department of Mechanical Engineering

P.O. Box 11000, FI-00076 AALTO

heikki.remes@aalto.fi, anssi.karttunen@aalto.fi 


\section{Appendix: Finite element calculation (Abaqus) results}

1. Stress distributions on semi-infinite plate for double semi-circular notch and notch hole models of $(R / a=5)$. The notch-hole pairs are studied at notch-hole gaps of $d=2.5 a$, $5 a$, and $10 a$ in a consecutive order. A uniaxial tension is applied with $\sigma_{\infty x}=33.3 \mathrm{pa}$. The plate is modelled with the following dimensions:

Plate length $(L)=1000 a$, Plate height $(H)=500 a$.
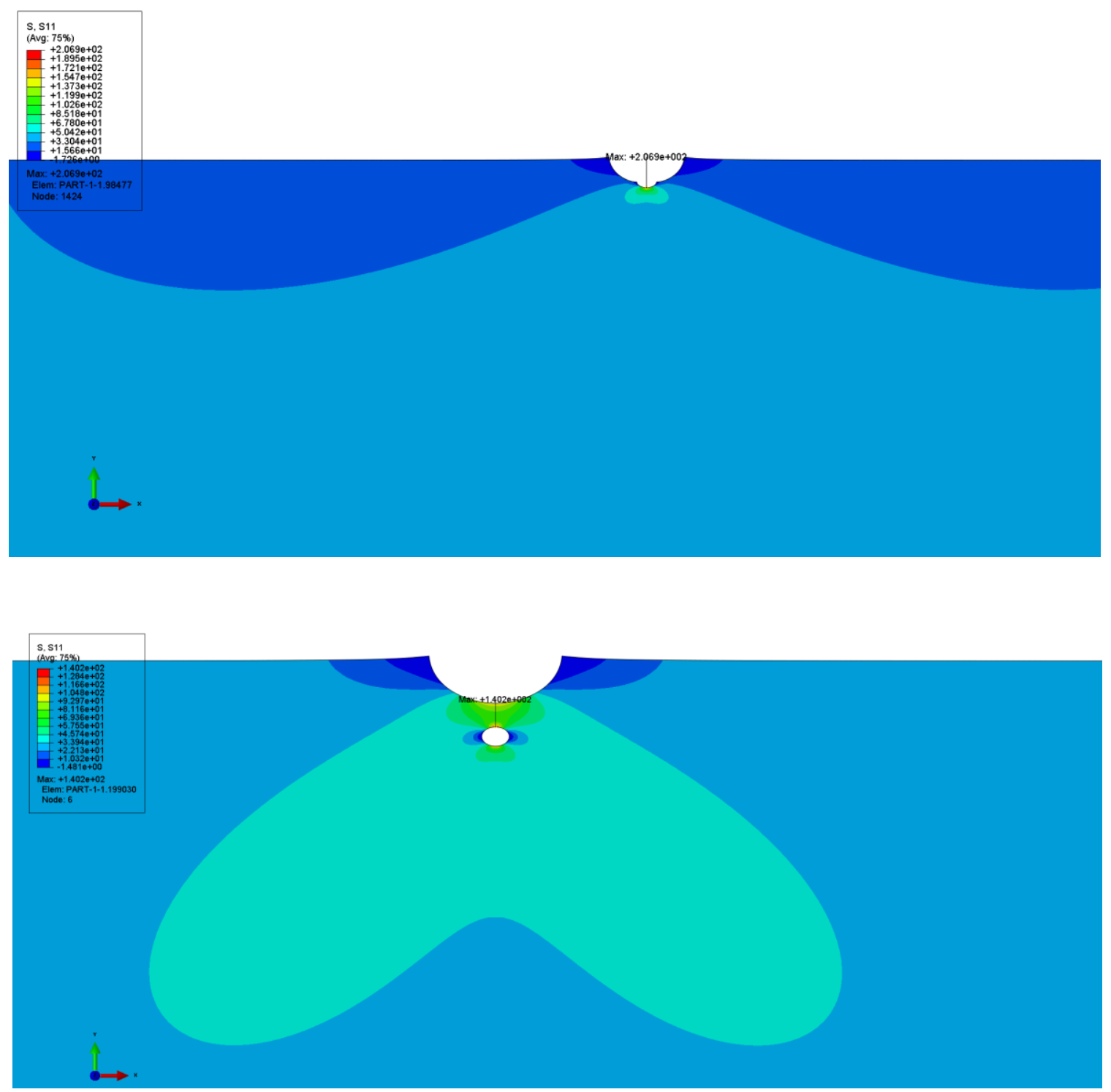

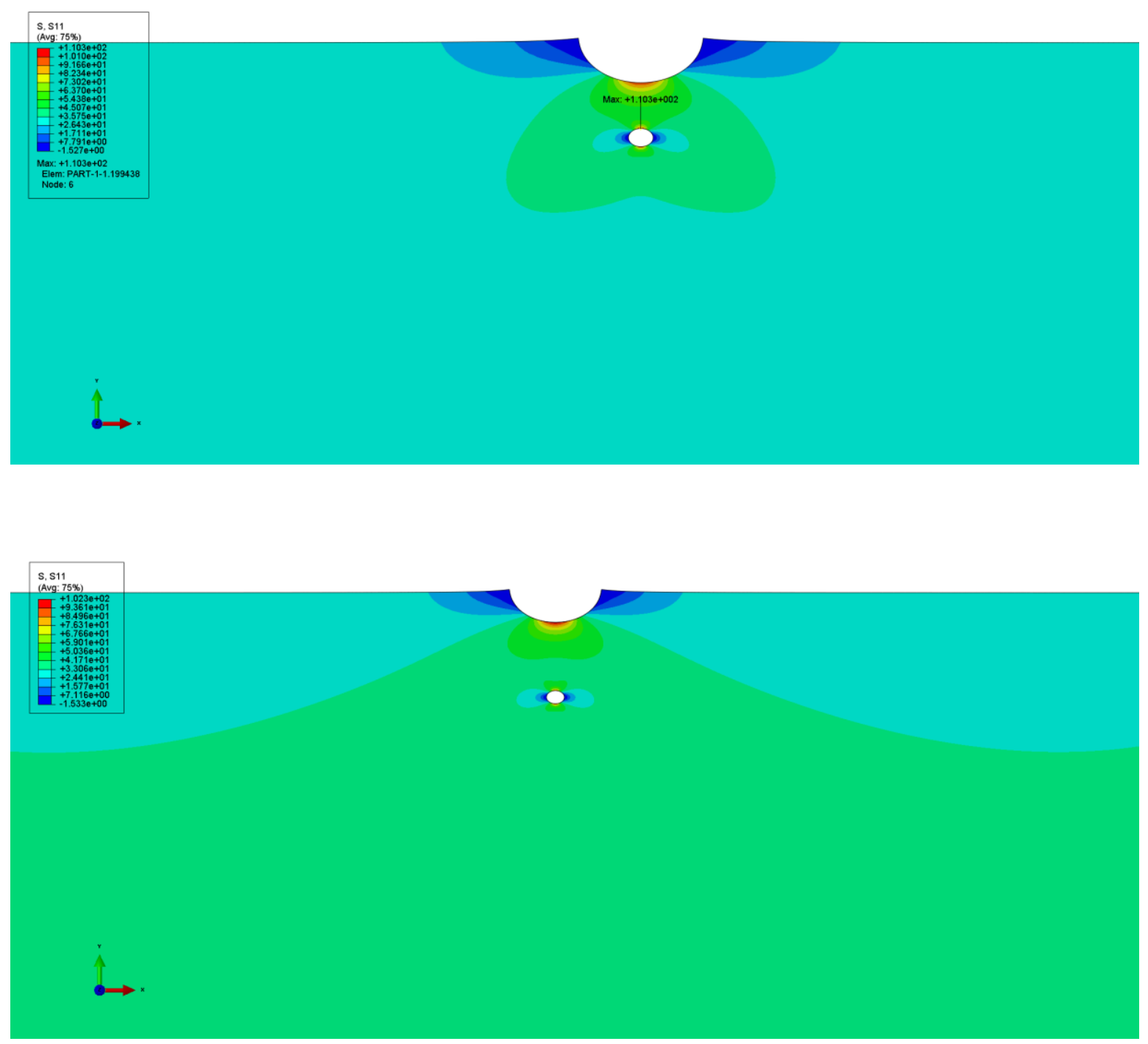
2. Stress distributions on semi-infinite plate for double semi-circular notch and notch hole models of $(R / a=10)$. The notch-hole pairs are studied at notch-hole gaps of $d=2.5 a, 5 a$, $10 a$ and $15 a$ in a consecutive order. A uniaxial tension is applied with $\sigma_{\infty x}=33.3 \mathrm{pa}$. The plate is modelled with the following dimensions:

Plate length $(L)=1000 a$, Plate height $(H)=500 a$.
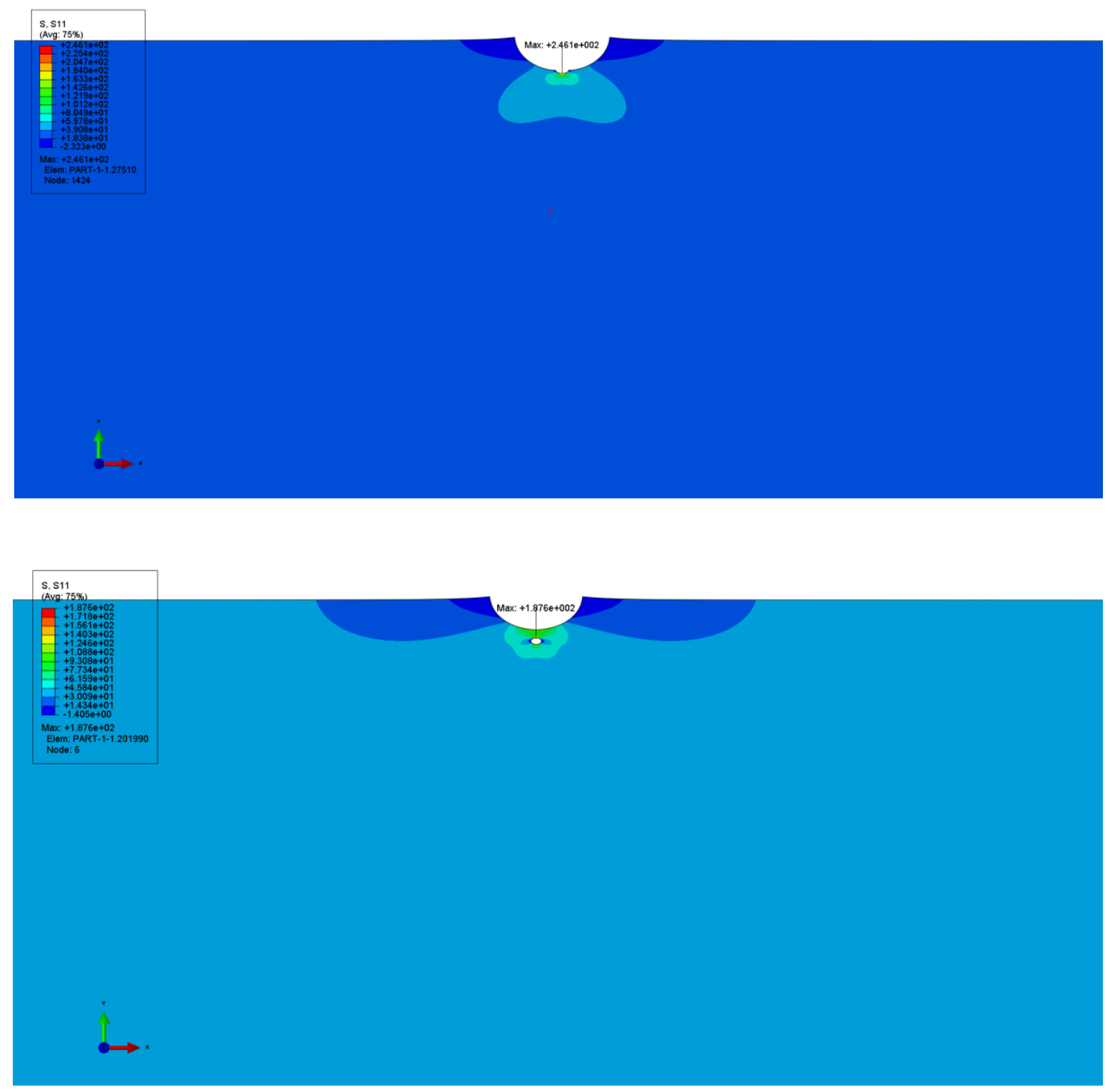

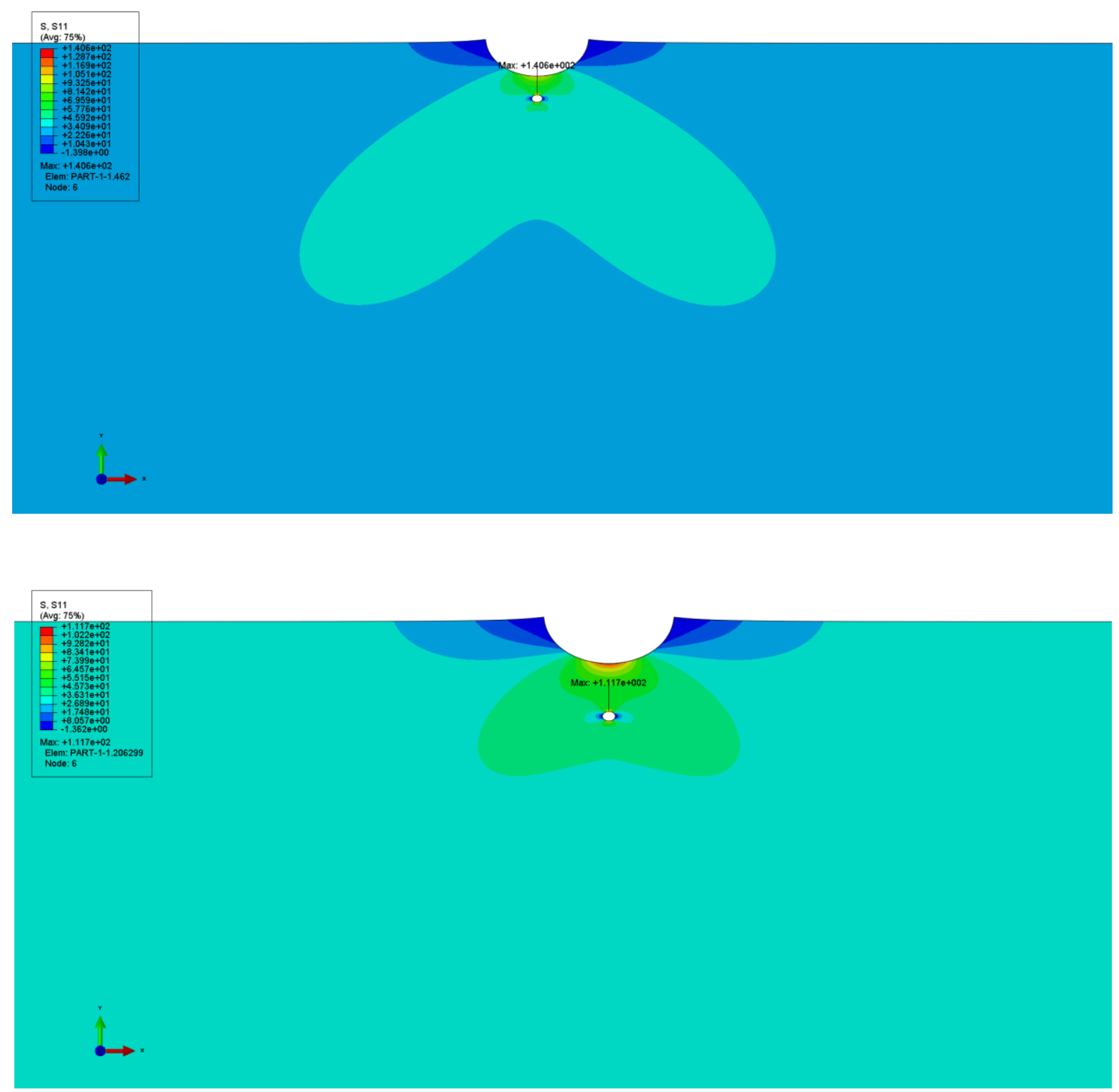
S. $\$ 11$

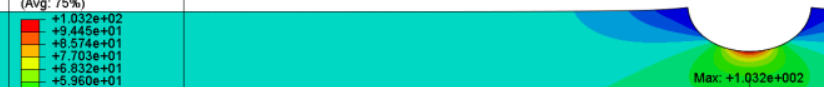

$\begin{aligned} &+4.218 e+0 \\ &-+3.347+01 \\ &-+2.476+01 \\ &+2.46+0\end{aligned}$

$+1.335 \mathrm{e}+00$
$\quad 1.377 \mathrm{e}+00$
-100

Max $+1.032 e+02$
Elem: PART $-1-1.208015$

1

$\underset{0}{\stackrel{A}{\longrightarrow}}$ 